\title{
Intermittency and multifractional Brownian character of geomagnetic time series
}

\author{
G. Consolini ${ }^{1}$, R. De Marco ${ }^{1}$, and P. De Michelis ${ }^{2}$ \\ ${ }^{1}$ INAF - Istituto di Astrofisica e Planetologia Spaziali, 00133 Rome, Italy \\ ${ }^{2}$ Istituto Nazionale di Geofisica e Vulcanologia, 00143 Rome, Italy \\ Correspondence to: G. Consolini (giuseppe.consolini@inaf.it)
}

Received: 30 October 2012 - Revised: 24 April 2013 - Accepted: 1 June 2013 - Published: 11 July 2013

\begin{abstract}
The Earth's magnetosphere exhibits a complex behavior in response to the solar wind conditions. This behavior, which is described in terms of mutifractional Brownian motions, could be the consequence of the occurrence of dynamical phase transitions. On the other hand, it has been shown that the dynamics of the geomagnetic signals is also characterized by intermittency at the smallest temporal scales. Here, we focus on the existence of a possible relationship in the geomagnetic time series between the multifractional Brownian motion character and the occurrence of intermittency. In detail, we investigate the multifractional nature of two long time series of the horizontal intensity of the Earth's magnetic field as measured at L'Aquila Geomagnetic Observatory during two years (2001 and 2008), which correspond to different conditions of solar activity. We propose a possible double origin of the intermittent character of the small-scale magnetic field fluctuations, which is related to both the multifractional nature of the geomagnetic field and the intermittent character of the disturbance level. Our results suggest a more complex nature of the geomagnetic response to solar wind changes than previously thought.
\end{abstract}

\section{Introduction}

The Earth's magnetosphere, which is the region of space where the geomagnetic field is confined by the solar wind, can be described as a nonequilibrium nonlinear complex system. Its complex dynamics, which results from the superposition of the internal dynamics and the external driving due to the changes of solar wind conditions, has been widely investigated during the past two decades. Several studies have been devoted to examine the occurrence of chaos, turbulence and criticality during geomagnetic storms and substorms, which are the most important manifestations of the magnetospheric activity (e.g., Klimas et al., 1996, 2000; Consolini and Chang, 2001; Dobias and Wanliss, 2009). These studies have clearly evidenced that the magnetospheric evolution is characterized by a near-criticality dynamics, which manifests both in the scale-invariant nature of magnetotail relaxation events and fractal properties of geomagnetic time series.

It has been found that the fractal character of several geomagnetic time series, for example those relative to the geomagnetic indices AE, Dst and SYM-H, changes during magnetic storms and substorms (Uritsky and Pudovkin, 1998; Wanliss, 2005; Balasis et al., 2006; Wanliss and Dobias, 2007; Dobias and Wanliss, 2009). In particular, it has been noticed that the self-similarity properties of these time series change when an intense magnetospheric/geomagnetic activity event approaches. For instance, Balasis et al. (2006) have shown the occurrence of an interesting transition from the anti-persistent to the persistent character in the fluctuations of Dst index, which closely corresponds to a transition from a regime of quiet to one of high magnetospheric activity, characterized by the occurrence of intense geomagnetic storms. A similar behavior has been also documented in the case of the auroral electrojet (AE) index (e.g., Uritsky and Pudovkin, 1998). This result suggests that the magnetospheric dynamics may arise from a combination of solar wind changes and internal magnetospheric activity. However, variations of the self-similarity features of geomagnetic indices have also been observed in the distribution functions of the smallscale increments (Consolini and De Michelis, 1998). These changes, which usually arise from nonequilibrium dynamical phase transitions occurring commonly at the onset of and during magnetic storms and substorms, have been interpreted 
and discussed in a more general scenario, involving a near forced and/or self-organized criticality (FSOC) dynamics (Consolini, 1997; Uritsky and Pudovkin, 1998; Chang, 1999; Chang et al., 2003; Consolini and Chang, 2001; Consolini and De Michelis, 2002; Wanliss and Weygand, 2007; Wanliss and Uritsky, 2010).

Another observable property of the magnetospheric dynamics, which is documented in literature, is the intermittent character of geomagnetic time series, i.e., the anomalous scaling of small-scale increments (fluctuations) and the presence of a multifractal character (see, e.g., Consolini et al., 1996; Consolini and De Michelis, 1998; Kovács et al., 2001; Wanliss et al., 2005; Consolini and De Michelis, 2011, etc.). This intermittent character seems to be a common property of magnetospheric dynamics that is generally observed in both the time series of geomagnetic indices and in situ magnetospheric and ground-based magnetic field measurements. The origin of this intermittent and multifractal character has been interpreted as a direct consequence of dynamical changes and turbulent dynamics. In this framework, Dobias and Wanliss (2009) recently noticed that the intermittent character observed during magnetic substorms and storms displays similar features when it is investigated in terms of fractal processes. This observation suggests that during magnetic storms and substorms there are common dynamical processes at the basis of the critical behavior of the Earth's magnetospheric dynamics in response to solar wind changes (Dobias and Wanliss, 2009).

The aforementioned different features seem reasonably to suggest that the nature of the geomagnetic time series, and in particular of the geomagnetic indices, may be similar to those of multifractional Brownian motion $(\mathrm{mBm})$ (Muniandy and Lim, 2001), where the scaling features change in time. In this case, we can legitimately ask what the link is between the observed intermittent and multifractal character of geomagnetic signal and its possible multifractional Brownian nature.

This paper is organized as follows. At first, a brief summary of the main properties of fractional and multifractional Brownian motions are described. Following this, the relationship between intermittency and $\mathrm{mBm}$ in geomagnetic time series is analyzed. Finally, the obtained results are summarized and the implications of the findings are discussed.

\section{Fractional and multifractional Brownian motion: some general features}

Fractional Brownian motion (fBm), also called fractal Brownian motion, was introduced by Mandelbrot and Van Ness (1968) as a natural extension of ordinary Brownian motion. A $\mathrm{fBm}$ has the property of being statistically self-similar, which means that any portion of it can be viewed, from a statistical point of view, as a scaled version of a larger part of the same process. Unlike the classical (also known as ordinary or standard) Brownian motion, which is characterized by independent increments, the $\mathrm{fBm}$ has a non-Markovian character, which is manifested in the correlation between past and future increments leading to persistent or anti-persistent behavior.

According to Mandelbrot and Van Ness (1968) the simplest way of defining a $\mathrm{fBm} B_{H}(t)$ is based on a modified fractional Weyl integral,

$$
\begin{aligned}
B_{H}(t)= & \frac{1}{\Gamma\left(H+\frac{1}{2}\right)}\left\{\int_{-\infty}^{0}\left[(t-s)^{H-\frac{1}{2}}-(-s)^{H-\frac{1}{2}}\right]\right. \\
& \left.\times \mathrm{d} B(s)+\int_{0}^{t}(t-s)^{H-\frac{1}{2}} \mathrm{~d} B(s)\right\},
\end{aligned}
$$

where $\Gamma(x)$ is the Euler Gamma function, and $H$ is the Hurst exponent (sometimes also called Hölder exponent), which varies in the interval $(0,1)$. The main features of the fBms are (i) a zero mean displacement,

$\left\langle B_{H}(t)\right\rangle=0$,

where \langle\rangle denotes the ensemble average and (ii) a timeincreasing variance of increments,

$$
\left\langle\left[\delta B_{H}(s)\right]^{2}\right\rangle \propto|s|^{2 H},
$$

where $\delta B_{H}(s)=B_{H}(t+s)-B_{H}(t)$ is the increment at the timescale $s$. Classical Brownian motion, which is characterized by time-independent increments, is recovered for $H=\frac{1}{2}$. Conversely, the increments are negatively correlated (i.e., to a positive/negative fluctuation it follows a negative/positive fluctuation with a higher probability) when $0<H<1 / 2$, and they are positively correlated when $1 / 2<$ $H<1$. Moreover, the increments of $\mathrm{fBm}$ are stationary and self-similar with the parameter $H$ :

$\left\{\delta B_{H}(s)\right\} \triangleq\left\{\lambda^{-H} \delta B_{H}(\lambda s)\right\}$,

where $\{X\} \triangleq$ distribution functions.

The fBms, being self-similar processes, allow us to conveniently describe irregular signals, which arise from several real situations. However, the pointwise regularity of a $\mathrm{fBm}$ is the same along its path, and this property is sometimes undesired, restricting the application field. For this reason, in the second half of the 1990s the definition of fBm, characterized by a global value of $H$, was generalized about the case in which $H$ is no longer independent of time, but a function of it. Consequently, in the generalization of $\mathrm{fBm}$, i.e., mBm (Peltier and Lévy-Vehel, 1995; Benassi et al., 1997), the Hurst exponent $H$ depends on time, and all the features of fBms are valid only locally. Among the different definitions of $\mathrm{mBms}$ one of them, based on the fractional Weyl integral representation, is easily obtained from Eq. (1) considering, contrary to what happens for $\mathrm{fBm}$, a time-dependent Hurst exponent $H(t)$. Thus, the increments of a $\mathrm{mBm}$ are no longer stationary and the process is no longer globally self-similar. It 


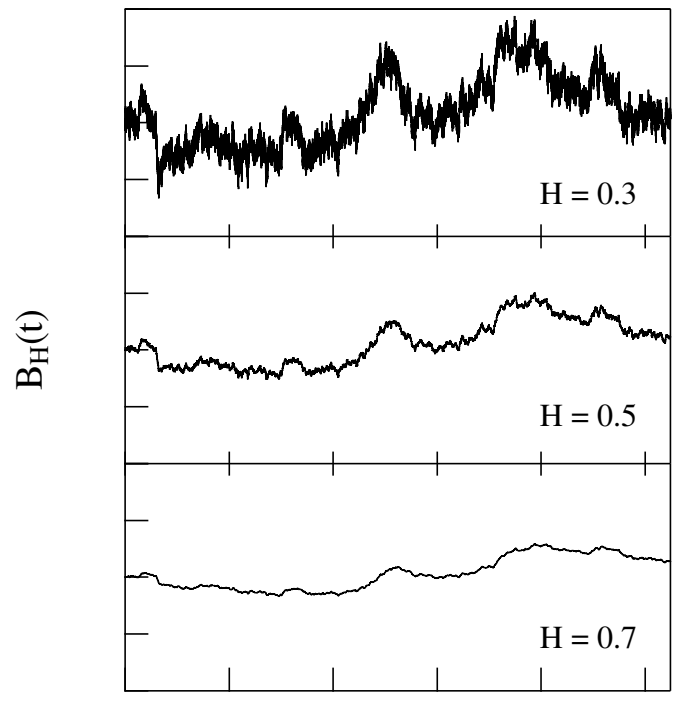

$\mathrm{t}$

Fig. 1. Three samples of $\mathrm{fBm}$ characterized by different values of the Hurst exponent $H$. The fBm samples are generated from the algorithm described in Benassi et al. (1997) with the same sequence of random numbers, so as to emphasize the differences in terms of fluctuations.

is possible to use a simpler representation of a $\operatorname{mBm} X_{H(t)}(t)$ based on a Riemann-Liouville fractional integral (RLMBM) (Muniandy and Lim, 2001),

$X_{H(t)}(t)=\frac{1}{\Gamma\left(H(t)+\frac{1}{2}\right)} \int_{0}^{t}(t-s)^{H(t)-\frac{1}{2}} \mathrm{~d} B(s)$,

where we use the notation $X_{H(t)}(t)$, instead of $B_{H}(t)$, to indicate a multifractional Brownian motion.

Starting from this definition, a direct scheme to generate a $\mathrm{mBm}$ has been implemented (Benassi et al., 1997; Muniandy and Lim, 2001). This scheme uses uncorrelated unit variance noise, i.e.,

$X_{H\left(t_{j}\right)}\left(t_{j}\right)=\sum_{i=1}^{j}\left(\frac{\eta_{i}}{\sqrt{\Delta t}}\right) w_{j-i+1} \Delta t$,

where $\Delta t=1 /(N-1), N$ equals the length of the series, $\eta_{i}$ is a unit variance Gaussian white noise with zero mean, and $w_{i}$ is a weight function (see Muniandy and Lim, 2001 for more details) defined as

$w_{i}=\frac{1}{\Gamma\left(H+\frac{1}{2}\right)}\left[\frac{t_{i}^{2 H}-\left(t_{i}-\Delta t\right)^{2 H}}{2 H \Delta t}\right]^{1 / 2}$.

We emphasize that synthetic $\mathrm{mBm}$ generated by means of RLMBM approximation (Eq. 6) results from the addition of weighted random values.

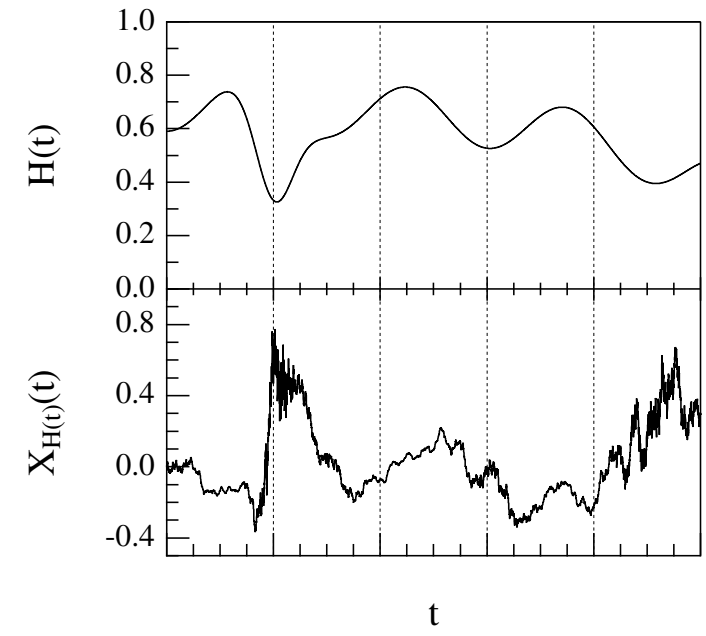

Fig. 2. A sample of $\mathrm{mBm}$ (lower panel), generated according to the procedure described in Muniandy and Lim (2001) with a timevarying Hurst/Hölder exponent $H(t)$ (upper panel). Note the nonstationary character of the fluctuations.

Figure 1 shows some samples of fBms, characterized by different values of the Hurst exponent, while Fig. 2 displays a sample of $\mathrm{mBm}$. These samples are generated from algorithms described in Benassi et al. (1997) and Muniandy and Lim (2001), respectively. As it can be seen from the plots, the $\mathrm{fBm}$ and $\mathrm{mBm}$ fluctuations have a different character. Indeed, while the $\mathrm{fBm}$ fluctuations are stationary in amplitude, the $\mathrm{mBm}$ fluctuations exhibit a clearly nonstationary character, and become highly relevant during those time intervals which are characterized by very low Hurst/Hölder exponent values. These two properties (nonstationarity and dependence on $H$ values) of the $\mathrm{mBm}$ fluctuations are confirmed by looking at the trend of the local variance $\sigma_{\mathrm{loc}}^{2}(t)$ (computed on a moving window of 101 points in the case of a $\mathrm{mBm}$ synthetic signal) as a function of the local Hurst exponent (Fig. 3) and analyzing simultaneously the shape of the probability density functions (PDFs) of $\mathrm{fBm}$ and $\mathrm{mBm}$ increments, $\delta B_{H}(\tau)$ and $\delta X_{H(t)}(\tau)$, respectively. Indeed, Fig. 3 clearly shows a decrease of the local signal variance $\sigma_{\mathrm{loc}}^{2}(t)$ with the local Hurst/Hölder exponent values, while Fig. 4 shows the nonGaussian shape of the PDFs for the set of fBms and $\mathrm{mBm}$, reported in Figs. 1 and 2. More exactly, Fig. 4 reports the PDFs for the smallest scale $(\tau=1)$ increments $\delta B_{H}(\tau)$ and $\delta X_{H(t)}(\tau)$, computed using synthetic signals of more than $5 \times 10^{5}$ points. The increments have been rescaled to unit variance for comparison. The nonstationary nature of the $\mathrm{mBm}$ increments (fluctuations) manifests itself by a significant departure of its PDF from the Gaussian shape. The $\mathrm{mBm}$ PDF is clearly leptokurtotic and its shape depends on the increment timescale $\tau$, as clearly demonstrated by the dependence of the kurtosis $\kappa$ of the $\mathrm{mBm}$ increments $\delta X_{H(t)}(\tau)$ on the timescale $\tau$ (see Fig. 5). This means that we are not capable of constructing a master scale-invariant shape of the 


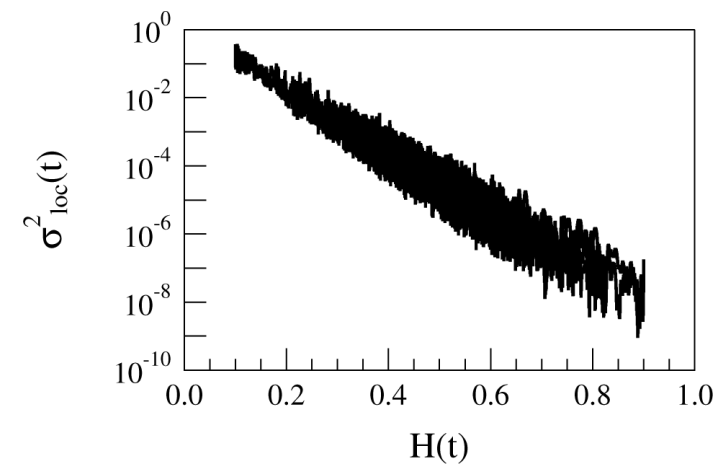

Fig. 3. Plot of the local variance $\sigma_{\text {loc }}^{2}(t)$ versus the local Hurst/Hölder exponent $H(t)$.

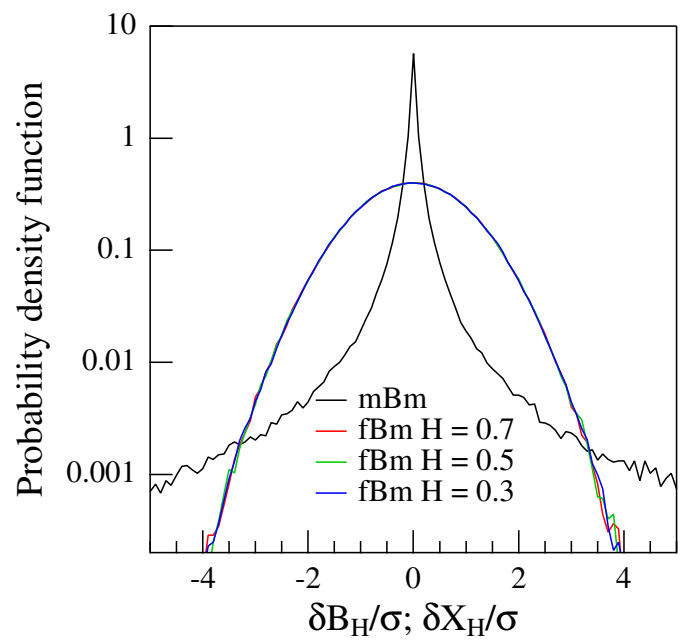

Fig. 4. Comparison among the PDFs of variance-normalized increments, $\delta B_{H}(\tau)$ and $\delta X_{H(t)}(\tau)$, of the $\mathrm{mBm}$ and $\mathrm{fBms}$ reported in previous figures. The shape of the function for the $\mathrm{fBms}$ is the same for all $H$ values.

increment PDFs at different timescales $\tau$, using a single scaling exponent. This is the evidence of the intermittent character of $\mathrm{mBm}$, which is the counterpart of the nonstationarity of $H(t)$.

\section{Data description and analysis}

To investigate the link between the intermittent character of geomagnetic time series and their multifractional nature, we consider two sets of data relative to the geomagnetic measurements of the horizontal intensity $(\mathcal{H})$ of the Earth's magnetic field recorded during two distinct years: the year 2001 and the year 2008, which correspond to periods of maximum and minimum solar activity, respectively. Data refer to magnetic field measurements recorded at L'Aquila Geomagnetic Observatory, which is part of INTERMAGNET program, whose objective is to establish a global network

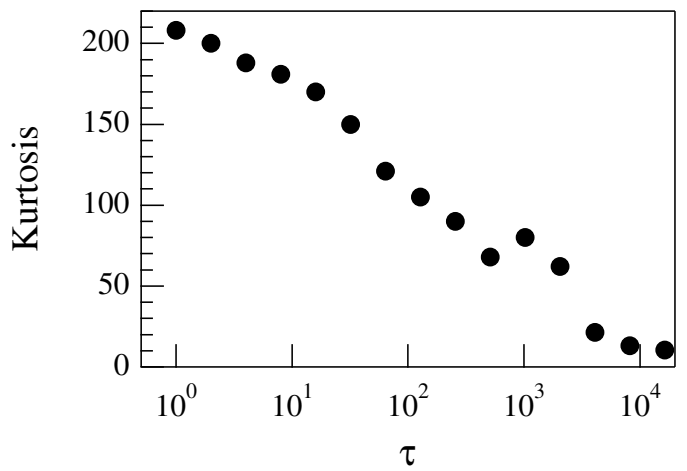

Fig. 5. Dependence of the kurtosis of the synthetic $\mathrm{mBm}$ increments $\delta X_{H(t)}(\tau)$ on the timescale $\tau$.

of co-operating high-quality digital magnetic observatories. Therefore, we make use of magnetic field recordings obtained by a permanent observatory fulfilling the same international standard of those used for constructing most of the commonly used geomagnetic indices. In particular, the data used in this standard are characterized by a sampling rate of $1 \mathrm{~min}$ and a resolution of $0.1 \mathrm{nT}$, which is higher than the usual resolution of geomagnetic indices, allowing a better investigation of small-scale fluctuations. Furthermore, these data, which are obtained from a geomagnetic observatory at mid-latitude (Lat. $42^{\circ} 23^{\prime} \mathrm{N}$, Long. $13^{\circ} 19^{\prime} \mathrm{E}$ ) located in the center of Italy, well represent the characteristic scenario in the evolution of geomagnetic field, and the selected magnetic field component is reflective of the Earth's space environment providing important information about the state of geomagnetic activity. Indeed, the competing balance between the Earth's intrinsic magnetic field and solar wind dynamical changes drives much of the variations of the Earth's space environment that are observed as magnetic field fluctuations at short timescales at ground level in our time series. Thus, our dataset can be considered to contain information on lowlatitude geomagnetic disturbances.

Figure 6 reports the two datasets of geomagnetic measurements during the two activity periods. At first glance, we immediately notice the different character and amplitude of the magnetic field fluctuations, which is due to the different solar activity level. From a statistical point of view, the fluctuations $(\delta \mathcal{H}=\mathcal{H}-\langle\mathcal{H}\rangle)$ of these two datasets are characterized by a large ratio of the variances, $\sigma_{2001}^{2} / \sigma_{2008}^{2} \sim 5.3$, and non-Gaussian distribution functions (here not shown), which show a more skewed $\left(\gamma_{2001}^{1} \simeq-2.8\right.$ and $\gamma_{2008}^{1} \simeq-0.9$, where $\gamma^{1}$ is the sample skewness) and kurtotic ( $\kappa_{2001} \simeq 18$ and $\kappa_{2008} \simeq 6$, where $\kappa$ is the sample kurtosis) character in the case of the 2001 dataset. Furthermore, there is a slight difference in the mean value of the horizontal intensity $\mathcal{H}$ of the Earth's magnetic field $(\Delta\langle\mathcal{H}\rangle \sim 90 \mathrm{nT})$, which is due to the secular variation of the field. 

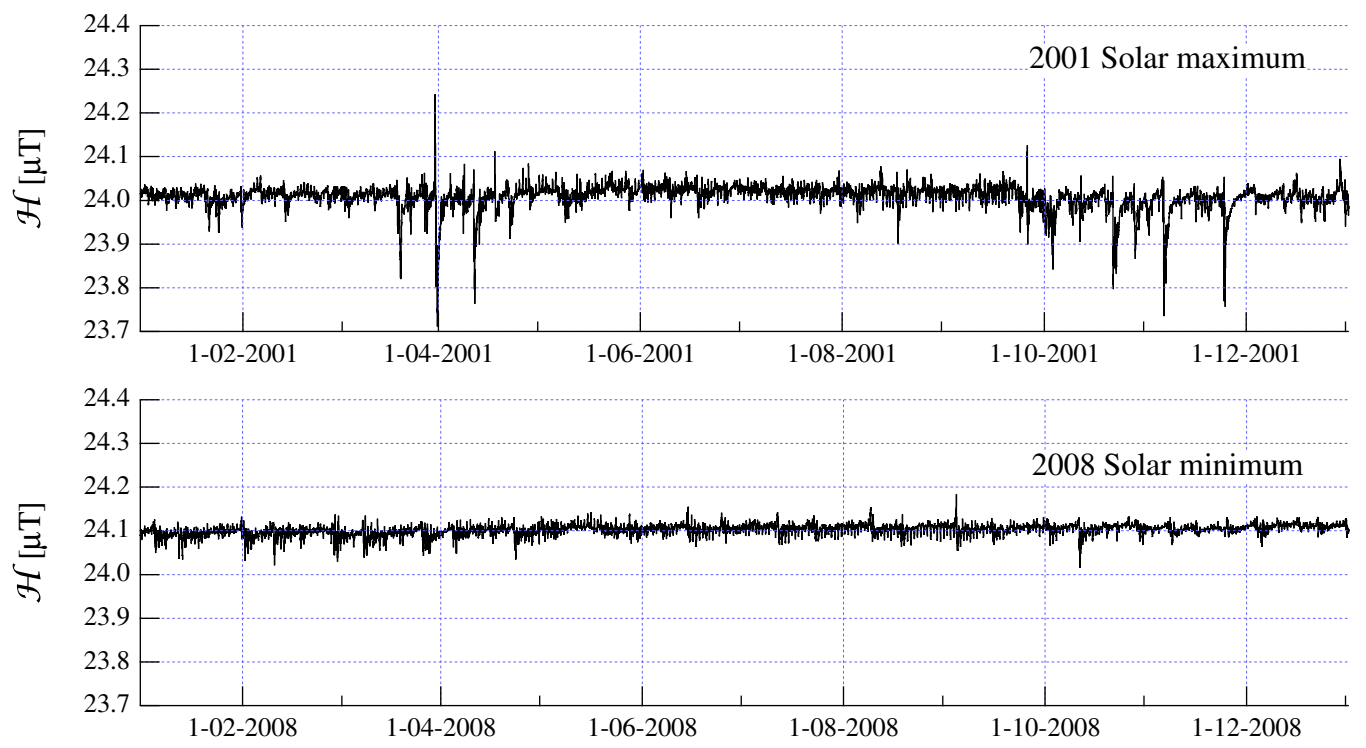

Fig. 6. The geomagnetic field horizontal intensity $\mathcal{H}$, as recorded at L'Aquila Geomagnetic Observatory (Italy) in 2001 (upper panel) and 2008 (lower panel).

To analyze the multifractional character of the geomagnetic time series, the first step of our analysis is to compute the power spectral density $S(f)$ of the magnetic field fluctuations by applying the standard Fourier spectral analysis. Figure 7 shows the PSDs obtained for the two selected time intervals. Both PSDs exhibit the same behavior at low and high frequencies displaying characteristic frequencies in correspondence with the daily variation $\left(f \sim 0.69 \times 10^{-3} \mathrm{~min}^{-1}\right)$ and its first harmonics. In addition, the PSDs at low frequencies (typically below $f \sim 0.006 \mathrm{~min}^{-1}$ ) are consistent with a power law spectrum $\left(S(f) \sim f^{-\alpha}\right)$ characterized by a spectral exponent $\alpha \sim 1.0 / 1.3$, while at the highest frequencies (above $f \sim 0.006 \mathrm{~min}^{-1}$ ) we observe a steeper power law spectrum, with a spectral exponent equal to $\alpha \sim 2.3$. This result suggests that the character of large- and small-scale fluctuations may be due to different physical processes. Furthermore, taking account of the relationship existing in the case of $\mathrm{fBm}$ between the Hurst exponent $H$ and the slope $\alpha$ of the PSD $(\alpha=2 H+1)$ existing for a $\mathrm{fBm}$, we can estimate the value of the average $H$ exponent for the two datasets. In both cases we obtain a value of $H \sim 0.65$, which suggests that overall character of the signals is consistent with persistent motions. The observed $H$ value, the $1 / f$ nature of the spectra and the persistent character at these timescales may be the counterpart of a possible critical dynamics (Woodard et al., 2007) in the magnetospheric response to solar wind changes as discussed in several works (see, e.g., Consolini and Chang, 2001). Nevertheless, at the present stage on the basis of our analysis this has to be considered only as a speculation; other possible explanations exist for the observed persistent character, for instance the contribution of regular variations (e.g., the daily variation $S_{q}$ ) to magnetic field measurements.
To fully characterize the differences among the fluctuations in the two datasets, we evaluate the kurtosis $\kappa$ of the magnetic field increments, $\delta \mathcal{H}(\tau)=\mathcal{H}(t+\tau)-\mathcal{H}(t)$, at different timescales $\tau$. Kurtosis is indeed a measure of whether the data-fluctuation PDFs are peaked or flat relative to a normal distribution. That is, datasets with high kurtosis have PDFs of fluctuations (or increments) that are characterized by a distinct peak near the most probable value and heavy tails in comparison with the Gaussian distribution. Conversely, low kurtosis datasets show flat top PDFs. In our case (see Fig. 8), we get high values of kurtosis, which depend on the timescale $\tau$ in both the selected periods. This dependence is more evident in 2001 than 2008. It is also interesting to observe that the highest values of kurtosis associated with short timescale increments are concentrated in the year 2001. This suggests that they are related to the higher solar activity level, which causes the occurrence of large geomagnetic storms.

The second step of our analysis to investigate the multifractional character of our magnetic field time series is to evaluate the local Hurst/Hölder exponent $H_{\text {loc }}(t)$. The evaluation of local Hurst exponent is a nontrivial target, and for this reason different approaches have been proposed in the past years (e.g., Peng et al., 1994; Peltier and Lévy-Vehel, 1995; Muniandy and Lim, 2001; Chen et al., 2002; Alessio et al., 2002; Carbone et al., 2004; Keylock, 2010). One of the most accurate, fast and simple methods for nonstandard Gaussian multifractional Brownian motions is the detrending moving average (DMA) technique introduced by Alessio et al. (2002) and Carbone et al. (2004). This method, which is based on the analysis of the scaling features of the local standard deviation around a moving average, is quite simple and seems to be more accurate than other methods 


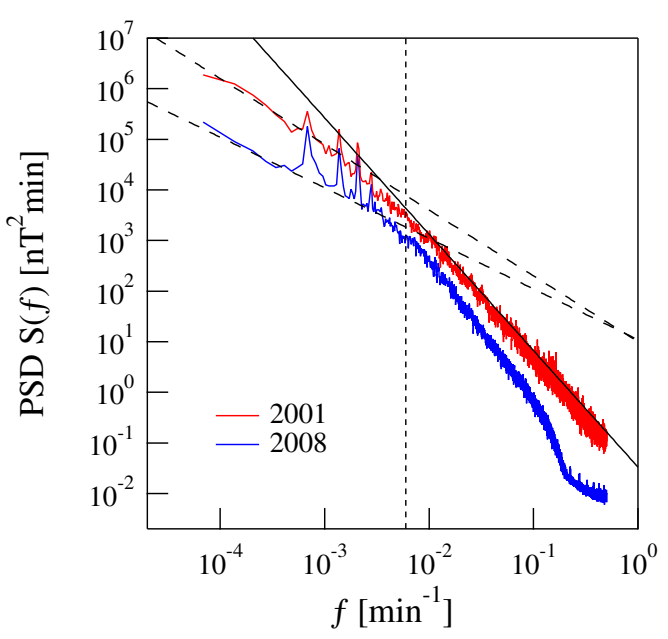

Fig. 7. The power spectral density $S(f)$ relative to the two selected time series. The vertical dashed line is at about $f=0.006 \mathrm{~min}^{-1}$. Solid and dashed lines are power law best fits.

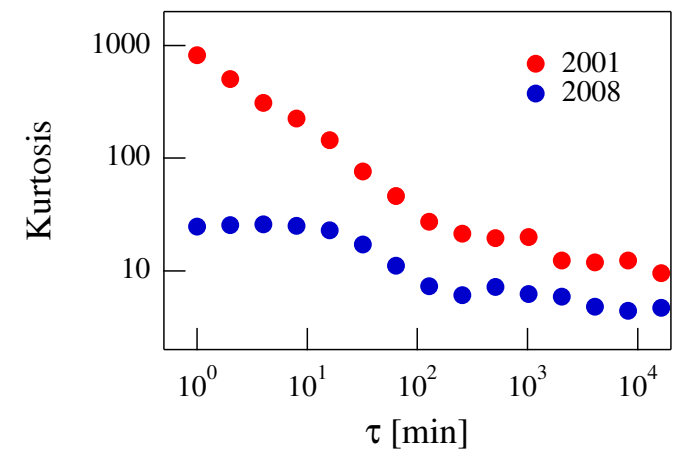

Fig. 8. The dependence of the kurtosis $\kappa$ of the magnetic field increments $(\delta \mathcal{H}(\tau))$ on the timescales $\tau$ for the two selected periods.

(Carbone et al., 2004). Indeed, the DMA algorithm is characterized by the better low-pass behavior of the moving average in comparison to polynomial filtering (Smith, 2003; Carbone et al., 2004). Nevertheless, we refer the reader to the literature on this matter (Alessio et al., 2002; Smith, 2003; Carbone et al., 2004) for a more detailed discussion on this point. The DMA technique consists in evaluating the scaling features of the quantity $\sigma_{\mathrm{DMA}}(n)$ defined as

$\sigma_{\mathrm{DMA}}(n)=\sqrt{\frac{1}{N_{\max }-n} \sum_{t=n}^{N_{\max }}\left[x(t)-\bar{x}_{n}(t)\right]^{2}}$,

where $\bar{x}_{n}(t)$ is the average on a moving time window of length $n$, for different values of the time window in the interval $\left[n, N_{\max }\right]$, and in calculating the scaling exponent of the $\sigma_{\mathrm{DMA}}(n)$, which is expected to scale as $\sigma_{\mathrm{DMA}}(n) \sim n^{H}$.

To test the reliability of this method, we apply the local estimation of Hurst exponent to the case of a synthetic signal of $\sim 5 \times 10^{5}$ points. In this case, the evaluation of $H_{\text {loc }}(t)$

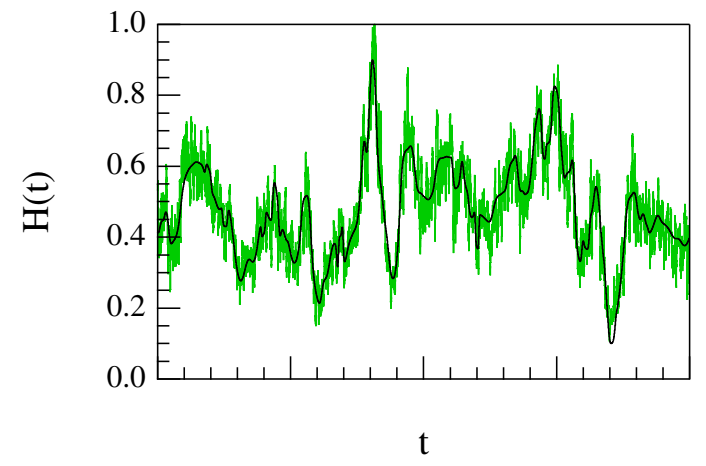

Fig. 9. A comparison between the actual local Hurst exponent (black line) and $H_{\text {loc }}(t)$ estimated using the DMA technique (green line). The average discrepancy between the true and DMAestimated local Hurst exponent is about $10 \%$.

is done using a moving window of 801 points and studying the scaling features for $n \in[2,100]$. The choice of a time window of 801 points has been done to ensure an optimal noise/signal ratio in determining the local exponent. Figure 9 shows the comparison between the actual $H(t)$ and the estimated $H_{\mathrm{loc}}(t)$ using the DMA technique for a short interval. The average precision in estimation of the $H_{\text {loc }}(t)$ is about $10 \%$ (i.e., $\left\langle\left|H_{\mathrm{loc}}(t)-H(t)\right| / H(t)\right\rangle \simeq 0.1$ ).

Furthermore, focusing our present study on the possible relationship between the multifractionality and intermittency, we analyze the fluctuations in the high-frequency domain, i.e., above the spectral break at about $f=6 \times$ $10^{-3} \mathrm{~min}^{-1}$, which corresponds to a temporal scale lower than $100 / 200 \mathrm{~min}$.

Figures 10 and 11 show the results of the local Hurst exponent $H_{\mathrm{loc}}(t)$, obtained using the DMA technique on a moving window of 801 points, in the case of the two selected time intervals. We notice that the average value $\left\langle H_{\text {loc }}(t)\right\rangle$ of local Hurst exponents is always higher than 0.5 $\left(\left\langle H_{\mathrm{loc}}(t)\right\rangle_{2001}=[0.75 \pm 0.16]\right.$ and $\left\langle H_{\mathrm{loc}}(t)\right\rangle_{2008}=[0.80 \pm$ $0.16])$, suggesting that the mean character of geomagnetic time series is well in agreement with that of a persistent $\mathrm{mBm}$, as already observed in geomagnetic index studies (e.g., Dobias and Wanliss, 2009), and with what is found in the case of a near-criticality dynamics (Woodard et al., 2007). However, we emphasize that the structure of the fluctuation field is sometimes not in agreement with a simple $\mathrm{mBm}$ being the value of the local Hurst exponent higher than 1. Furthermore, during periods characterized by intense disturbances (see, e.g., the period near the end of March 2001) the character of the fluctuation field seems to display a less persistent character. We underline that this behavior refers to fluctuations characterized by timescales below $100 \mathrm{~min}$, and that a possible origin of such less persistent character may be related to the rapid and impulsive variations of a web-like structure of filamentary currents flowing in the ionosphere. 


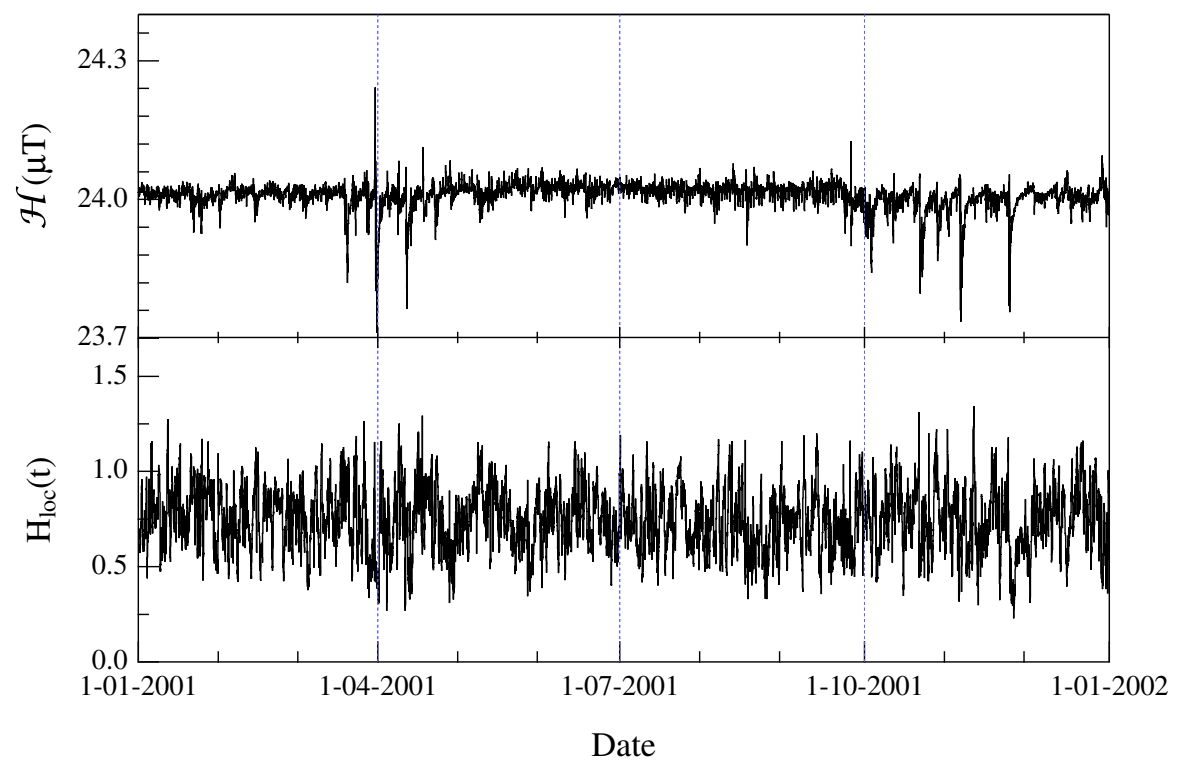

Fig. 10. The geomagnetic field $\mathcal{H}$ component, as measured at L'Aquila Geomagnetic Observatory (Italy) in 2001 (upper panel), and the corresponding local Hurst exponent $H_{\text {loc }}(t)$ (lower panel), computed using the DMA technique.

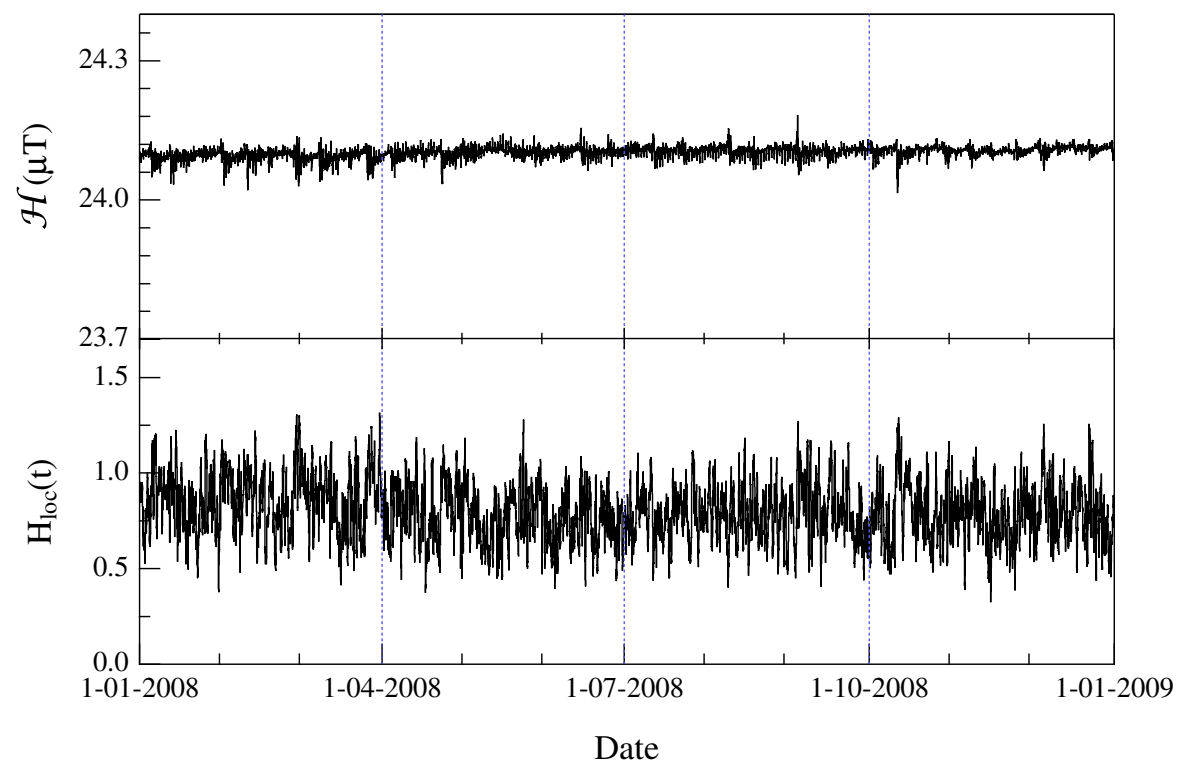

Fig. 11. The geomagnetic field $\mathcal{H}$ component, as measured at L'Aquila Geomagnetic Observatory (Italy) in 2008 (upper panel), and the corresponding local Hurst exponent $H_{\mathrm{loc}}(t)$ (lower panel), computed using the DMA technique.

Clearly, this point requires a different and more accurate analysis, which is outside the aim of this work.

As the third step in the analysis of the $\mathrm{mBm}$ character of geomagnetic time series, we compute the probability distribution functions of the local Hurst exponents $H_{\text {loc }}(t)$. These PDFs are reported in Fig. 12 for both the selected time periods. The two PDFs are completely equivalent and consistent with a Gaussian shape, showing only a small difference in the central value, $\left\langle H_{\text {loc }}\right\rangle$, which is shifted towards lower values of $H_{\text {loc }}$ for 2001. The observed difference $\left(\delta\left\langle H_{\text {loc }}\right\rangle=\right.$ $\left.\left\langle H_{\text {loc }}\right\rangle_{2008}-\left\langle H_{\text {loc }}\right\rangle_{2001}\right)$ is $\delta\left\langle H_{\text {loc }}\right\rangle=0.05$ in central value. This point towards a less persistent character of the fluctuations during the period of solar maximum, as a result of the more frequent occurrence of perturbed periods.

To better clarify the link between the intermittency and the multifractional nature of geomagnetic small-scale fluctuations, we investigate the relationship between the local Hurst exponent $H_{\mathrm{loc}}(t)$ and the local variance of the signal 


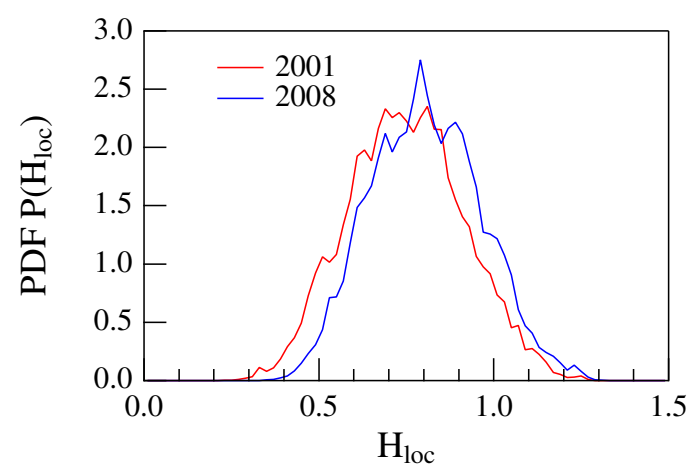

Fig. 12. The PDFs of the local Hurst exponent $H_{\text {loc }}$ computed for the two selected periods by means of the DMA technique.

$\sigma_{\mathrm{loc}}^{2}(t)$ for the two different datasets. The local variance is evaluated using a moving window of the same number of points as that used in DMA analysis, i.e., $N_{\text {points }}=801$. In addition, to reduce the effect of local trend at scales larger than the investigated ones, before evaluating the local variance, we detrend the signal in each window by a moving average technique on a scale larger than 101 points, i.e., on timescales larger than the maximum timescale investigated.

Figure 13 shows the occurrence plot of the dependence of the local Hurst exponent $H_{\text {loc }}$, computed by the DMA technique, on the corresponding local variance $\sigma_{\text {loc }}^{2}$ for the two time intervals here considered. $H_{\text {loc }}$ shows a tendency to increase with the increasing of $\sigma_{\text {loc }}^{2}$, although this positive correlation is more pronounced in 2008 (Pearson coefficient $r \sim 0.57$ ) than in 2001 (Pearson coefficient $r \sim 0.21$ ). However, this trend is different and opposite to that observed in the synthetic $\mathrm{mBm}$ signal generated using the algorithm described in Sect. 2, and displayed in Fig. 14 (Pearson coefficient $r \sim-0.88$ ).

The substantial discrepancy, observed between actual geomagnetic time series and synthetic $\mathrm{mBms}$, suggests that the intermittency of geomagnetic time series cannot be simply described as a consequence of their mutifractional nature. This different correlation character between $\sigma_{\text {loc }}^{2}$ and $H_{\text {loc }}$ suggests the existence of a double origin of intermittency, which is related to both their multifractional feature and fluctuation amplitudes.

To clarify this possible different origin of the intermittency, we can investigate the PDFs of the small-scale increments $\delta \mathcal{H}(\tau)$ (here $\tau=1 \mathrm{~min}$ ) of the geomagnetic field measurements, conditioned to the local Hurst exponent $H_{\text {loc }}$, and compare them with the corresponding conditioned PDFs of the small-scale increments of synthetic $\mathrm{mBm}$. In what follows, we identify these small-scale increments as $\delta X$, and the corresponding standard-deviation-normalized increments as $y=\delta X / \sigma$ for convenience. Furthermore, because the accuracy of the local Hurst index estimated using the DMA technique is within $10 \%$, to properly evaluate the PDFs of the small-scale increments for each choice of $H_{\text {loc }}$ we consider a window, characterized by a width of the same magnitude of the estimated precision, i.e., $\Delta H_{\mathrm{loc}} / H_{\mathrm{loc}} \sim 0.1$. In addition, for each $H_{\text {loc }}$-conditioned set of increments we evaluate the standard variation $\sigma\left(\delta X \mid H_{\text {loc }}\right)$ of the selected dataset. In this way we can study the dependence of $\sigma\left(\delta X \mid H_{\text {loc }}\right)$ on $H_{\text {loc }}$.

Figure 15 shows the conditioned PDFs $P\left(y \mid H_{\text {loc }}\right)$ of the standard-deviation-normalized small-scale $(1 \mathrm{~min})$ increments for the two selected time periods. A clearly different dependence of the shape of conditioned PDFs is observable in the two datasets. In detail, while for the year 2008 the shape of the conditioned PDFs looks quasi-independent of the value of $H_{\text {loc }}$, for the year 2001 a clear dependence is recovered. Indeed, the conditioned PDFs relative to the year 2001 increase their leptokurtotic character with the decrease of $H_{\text {loc }}$. This result is the signature of a different physical nature of small-scale increments relative to periods characterized by a lower value of $H_{\mathrm{loc}}$. A possible explanation of this different character of 2001/2008 conditioned PDFs could be the different level of solar activity and geomagnetic disturbances. Indeed, the more leptokurtotic character of the conditioned PDFs at low $H$ values in the year 2001 has to be related to the less persistent nature of very large fluctuations occurring during disturbed periods.

In Fig. 16 we report the conditioned PDFs $P\left(y \mid H_{\text {loc }}\right)$ obtained in the case of a synthetic $\mathrm{mBm}$ with a time-dependent local Hurst exponent $H_{\mathrm{loc}}(t) \in[0.1,0.9]$. The PDFs do not exhibit any substantial dependence of the shape on the value of $H_{\text {loc }}$. Furthermore, the shape of the conditioned PDFs is remarkably similar to that obtained evaluating the conditioned PDFs for the year 2008, which is characterized by a very low level of geomagnetic disturbance. Figure 17 provides a direct confirmation of this last point. In this figure we report the conditioned PDFs $P\left(y \mid H_{\mathrm{loc}}\right)$ with $H_{\mathrm{loc}}=0.5$ in the case of the two selected geomagnetic time series and the synthetic $\mathrm{mBm}$.

In the framework of fully developed fluid turbulence the departure from the Gaussian shape of the PDFs of smallscale velocity longitudinal increments is sometimes modeled by means of a superposition of Gaussian distribution characterized by variances distributed according to a log-normal distribution (Castaing et al., 1990). Analogously, we may think that the non-Gaussian shape of the small-timescale increments for fixed value of $H$ of the geomagnetic time series may be modeled using a similar approach. For instance, we can write

$$
P(\delta B \mid H)=\int p\left(\sigma^{2} \mid H\right) \frac{1}{\sqrt{2 \pi \sigma^{2}}} \exp \left(-\frac{\delta B^{2}}{2 \sigma^{2}}\right) \mathrm{d} \sigma^{2},
$$

where $p\left(\sigma^{2} \mid H\right)$ is the probability distribution function of the variances conditioned to the Hurst exponent. Consequently, the PDFs of the increments of the actual geomagnetic field measurements can be written as follows: 

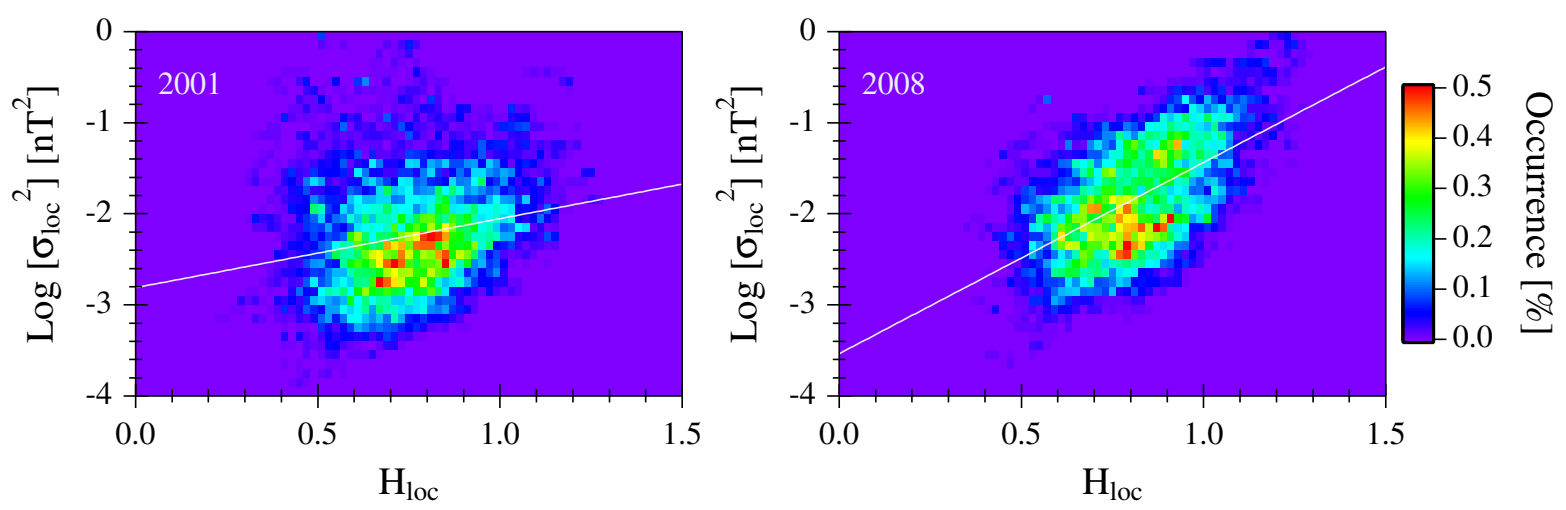

Fig. 13. The occurrence plot of the dependence of the local Hurst exponent $H_{\text {loc }}$, computed by the DMA technique, on the corresponding local variance $\sigma_{\text {loc }}^{2}$ for the year 2001 (left panel) and the year 2008 (right panel), respectively. The solid white line is a linear fit of the corresponding scatter plot, denoting a positive correlation between $\log _{10}\left[\sigma_{\text {loc }}^{2}\right]$ and $H_{\text {loc }}$. The significance of this positive correlation is more relevant for $2008(r \sim 0.57)$ than $2001(r \sim 0.21)$.

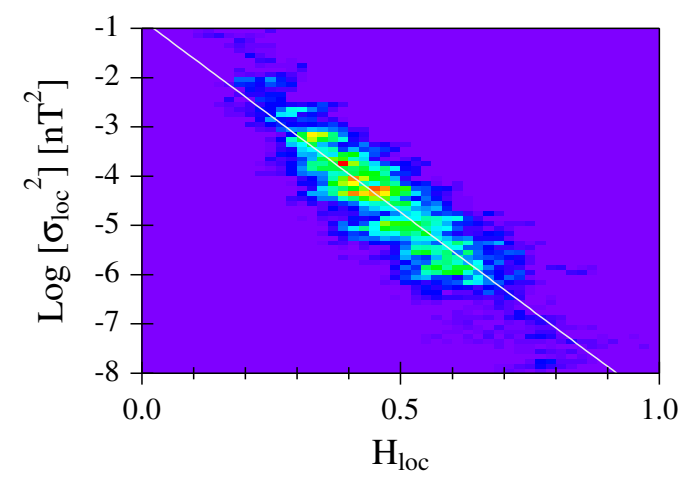

Fig. 14. The occurrence plot of the dependence of the local Hurst exponent $H_{\text {loc }}$, computed by the DMA technique, on the corresponding local variance $\sigma_{\text {loc }}^{2}$ for synthetic $\mathrm{mBm}$ signal. The solid white line is a linear fit of the corresponding scatter plot, denoting a clear negative correlation $(r \sim-0.88)$ between $\log _{10}\left[\sigma_{\text {loc }}^{2}\right]$ and $H_{\text {loc }}$. The used color palette is the same of Fig. 13.

$P(\delta B)=\int P(\delta B \mid H) p(H) \mathrm{d} H$,

$p(H)$ being the distribution function of the local Hurst exponents.

Last but not least, the more leptokurtotic character of the conditioned PDFs of increments of the geomagnetic field during high solar activity periods could reflect the different nature of the physical processes responsible for the geomagnetic disturbances. In detail, we can imagine that during these periods the nature of the geomagnetic perturbations and fluctuations may acquire a multiplicative character, which manifests in a significant departure of the conditioned PDFs from the shape obtained in the case of standard multifractional Brownian motions. This point could be a consequence of the
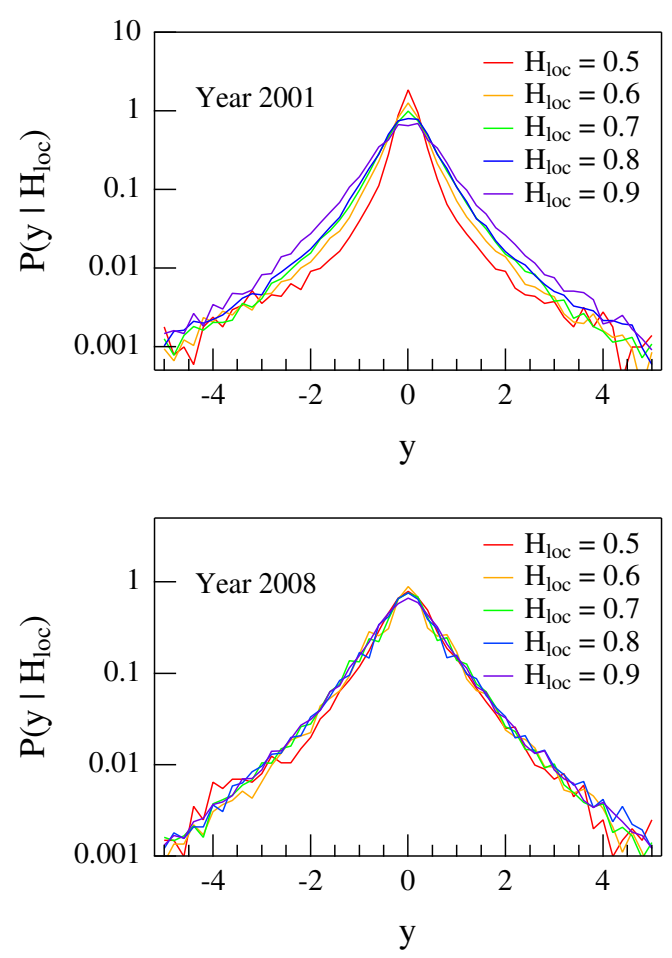

Fig. 15. The $H_{\text {loc }}$-conditioned probability distribution functions $P\left(y \mid H_{\mathrm{loc}}\right)$ of the standard-deviation-normalized small-scale ( $1 \mathrm{~min}$ ) increments for different values of $H_{\mathrm{loc}}$ in the range $[0.5,0.9]$. Upper/lower panel refers to year 2001/2008, respectively.

avalanching nature of magnetospheric dynamics (Consolini and Chang, 2001; Chang et al., 2003) during periods characterized by the occurrence of geomagnetic substorms and storms. 


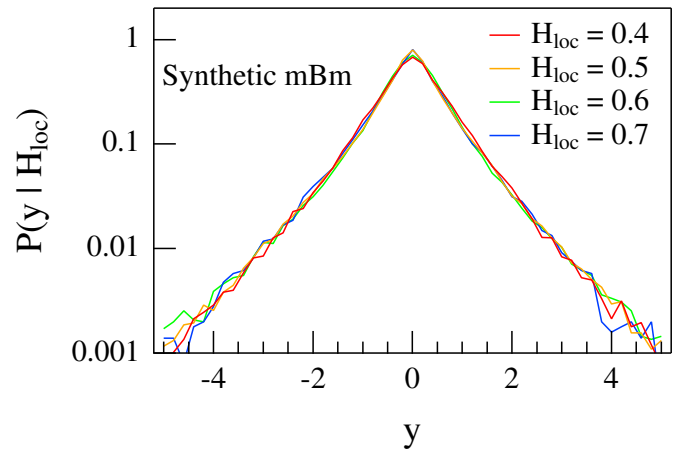

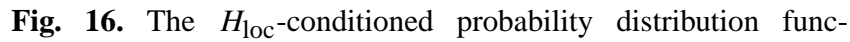
tions $P\left(y \mid H_{\text {loc }}\right)$ of the standard-deviation-normalized small-scale (1 min) increments for different values of $H_{\text {loc }}$ in the range [0.3, 0.7] in the case of synthetic $\mathrm{mBm}$ generated according to the algorithm described in Sect. 2.

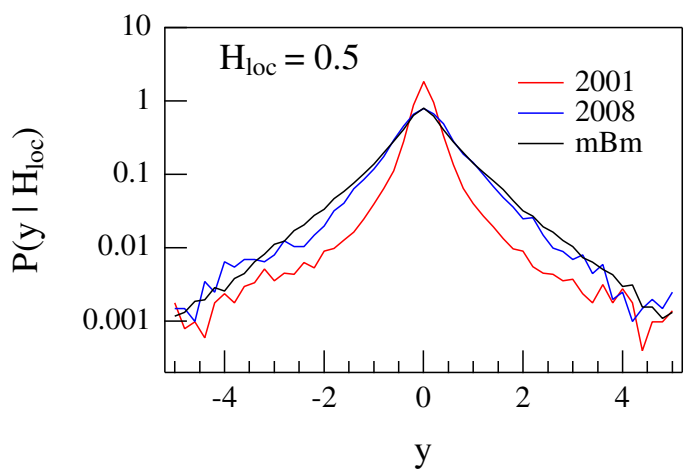

Fig. 17. Comparison of the $H_{\text {loc }}$-conditioned probability distribution functions $P\left(y \mid H_{\text {loc }}\right)$ of the standard-deviation-normalized small-scale $(1 \mathrm{~min})$ increments for $H_{\mathrm{loc}}=0.5$ in the case of the two selected geomagnetic time series and of the synthetic $\mathrm{mBm}$ generated according to the algorithm described in Sect. 2.

To conclude the characterization of the small-scale increments of conditioned PDFs, Fig. 18 shows the dependence of the standard deviation $\sigma\left(\delta X \mid H_{\text {loc }}\right)$ on $H_{\text {loc }}$ for the studied cases. A different behavior is recovered from the different cases here investigated. In particular, we detect a small positive correlation between $\sigma\left(\delta X \mid H_{\text {loc }}\right)$ and $H_{\text {loc }}$ for the year 2008, while in the other two cases (year 2001 and synthetic $\mathrm{mBm}$ signal $)$ we find that the standard deviation $\sigma\left(\delta X \mid H_{\text {loc }}\right)$ and $H_{\text {loc }}$ are clearly anti-correlated. The observed different behavior may suggest that the origin of intermittency in geomagnetic time series could be due to a more complex nature resulting from different competing processes, which are both of an internal magnetospheric origin and directly driven by solar wind disturbances. We will return to this point in the next section.

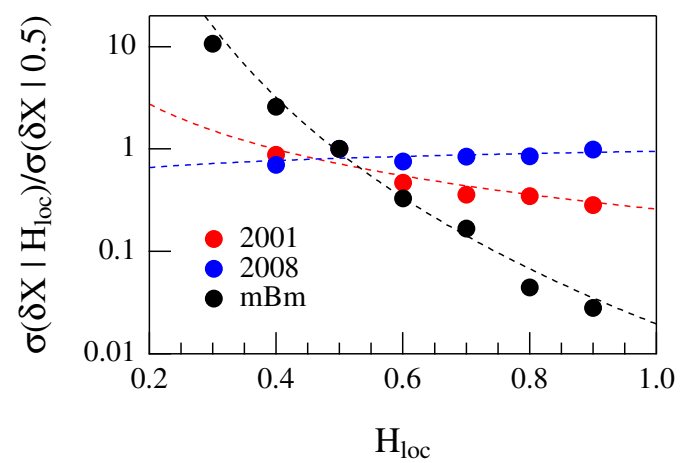

Fig. 18. Plot of the dependence of the standard deviation $\sigma(\delta X \mid$ $\left.H_{\text {loc }}\right)$ on $H_{\text {loc }}$. The standard deviation $\sigma\left(\delta X \mid H_{\text {loc }}\right)$ has been scaled to that corresponding to $H_{\mathrm{loc}}=0.5$ for convenience. Dashed lines are eye guides.

\section{Summary and conclusions}

We have focused our present work on the possible link between the intermittent character and the multifractional nature of the Earth's magnetic field fluctuations on small timescales. This study has been carried out by comparing actual recorded signals relative to different solar activity levels with synthetic mBms. The main results of our analysis can be summarized as follows:

1. The intermittent character of geomagnetic time series cannot be simply related to their multifractional features.

2. The high variability of solar wind activity level affects the multifractional character by changing the spectrum of local Hurst exponents. When the geomagnetic activity level increases, a decrease in the persistent character of geomagnetic fluctuations (increments) is observed.

3. The $H_{\mathrm{loc}}$-conditioned PDFs of the geomagnetic smalltimescale increments show a clear dependence on the geomagnetic activity level. Indeed, the shape of conditioned PDFs is independent of the $H_{\text {loc }}$ during solar minimum, while a clear dependence of the conditioned PDF shape on the $H_{\text {loc }}$ is found during solar maximum. This point is a direct consequence of the previous point as discussed in the previous section.

4. The observed shape of conditioned PDF during solar minimum resembles that of $\mathrm{mBm}$, while a considerable difference is observed in the case of solar maximum. This behavior is evident for the conditioned PDFs with $H_{\text {loc }}=0.5$, which are strongly affected by the geomagnetic disturbance level.

To outline some possible implications of our findings, we briefly discuss the scenario emerging from our analysis on 
the multifractional character of geomagnetic fluctuations. It is known that the Earth's magnetospheric dynamics is characterized by persistent dynamic correlations (e.g., Dobias and Wanliss, 2009) and near-criticality dynamics (e.g., Consolini, 2002; Wanliss and Uritsky, 2010), which are correlated features in self-organized critical systems in stationary conditions also away from the critical point (Woodard et al., 2007). Furthermore, the persistent character of the magnetospheric dynamics has been shown to undergo dynamical changes during magnetic storms and substorms (e.g., Wanliss, 2005; Balasis et al., 2006; Wanliss and Dobias, 2007). These points suggest that the more complex observed relation between the multifractional nature and the intermittent character of the geomagnetic fluctuations (see, e.g., Figs. 12, 13 and 14) may be the consequence of the nonstationarity conditions of the solar wind driving. This idea is corroborated by the high temporal variability of the increment variance, which has to be related to the variation in the solar wind magnetosphere coupling due to the changes of the solar wind conditions. In contrast, it seems to be very difficult to relate the nonstationary character of the solar wind driving to the less persistent character of geomagnetic field fluctuations during disturbed periods, which seems to be a peculiar property of ground-based magnetic field measurements with respect to geomagnetic indices, which show an increase of persistent dynamical correlations during disturbed periods (e.g., Dobias and Wanliss, 2009). A possible explanation of this different nature could be found in an inherent stochastic nature of the current patterns at the ionospheric level. Indeed, the less persistent nature of the geomagnetic fluctuations observed during magnetic storms could be due to the superposition of temporal and spatial fluctuations. The ground-based magnetic field measurements are the result of measurements that occur in different places on Earth; for this reason, they feel the effect of the local spatial structure of the ionospheric current system patterns. This is the main difference between the ground-based magnetic field measurements and the geomagnetic indices which differently provide global information on magnetospheric dynamics. Thus, we propose that the turbulent nature of the solar wind driving and the complex ionospheric current spatial pattern could be responsible for the more complex multifractional character of the geomagnetic fluctuations observed in our work, especially regarding the different dependence of signal variance on Hurst exponent with respect to simple synthetic $\mathrm{mBms}$. Clearly, the relation between these two different sources of the ground-based magnetic field disturbances is something that needs to be better investigated and clarified using much longer datasets and numerical simulations.

Acknowledgements. The results presented in this paper rely on the data collected at Geomagnetic Observatory of L'Aquila (Italy). We thank the Italian Istituto Nazionale di Geofisica e Vulcanologia (INGV), for supporting its operation, and INTERMAGNET for promoting high standards of geomagnetic observatory practice (http://www.intermagnet.org). This work was supported by the Italian Space Agency (ASI) under the ASI-INAF agreement no. I/022/10/0.

Edited by: J. Wanliss

Reviewed by: A. C.-L. Chian and one anonymous referee

\section{References}

Alessio, E., Carbone, A., Castelli, G., and Frappietro, V.: Secondorder moving average and scaling of stochastic time series, Eur. Phys. J. B, 27, 197-200, 2002.

Balasis, G., Daglis, I. A., Kapiris, P., Mandea, M., Vassiliadis, D., and Eftaxias, K.: From pre-storm activity to magnetic storms: a transition described in terms of fractal dynamics, Ann. Geophys., 24, 3557-3567, doi:10.5194/angeo-24-3557-2006, 2006.

Benassi, A., Jaffard, S., and Roux, D.: Elliptic Gaussian random processes, Rev. Mat. Iberoamericana, 13, 19-90, 1997.

Carbone, A., Castelli, G., and Stanley, H. E.: Time-dependent Hurst exponent in financial time series, Physica A, 344, 267-271, 2004.

Castaing, B., Gagne, Y., and Hopfinger, E. J.: Velocity probability density functions of high Reynolds number turbulence, Physica D, 46, 177-200, 1990.

Chang, T.: Self-organized criticality, multi-fractal spectra, sporadic localized reconnections and intermittent turbulence in magnetotail, Phys. Plasma, 6, 4137-4145, 1999.

Chang, T., Tam, S. W. Y., Wu, C. C., and Consolini, G.: Complexity, forced and/or self-organized criticality, and topological phase transitions in space plasmas, Space Sci. Rev., 107, 425445, 2003.

Chen, Z., Ivanov, P. Ch., Hu, K., and Stanley, H. E.: Effect of nonstationarities on detrended fluctuation analysis, Phys. Rev. E, 65, 041107, doi:10.1103/PhysRevE.65.041107, 2002.

Consolini, G.: Sandpile cellular automata and magnetospheric dynamics, in: Cosmic physics in the year 2000, edited by: Aiello, S., Iucci, N., Sironi, G., Treves, A., and Villante, U., SIF Conference Proc. 58, 123-126, 1997.

Consolini, G.: Self-Organized Criticality: A new paradigm for the magnetotail dynamics, Fractals, 10, 275-283, 2002.

Consolini, G. and Chang, T.: Magnetic field topology and criticality in geotail dynamics: relevance to substorm phenomena, Space Sci. Rev., 95, 309-321, 2001.

Consolini, G. and De Michelis, P.: Non-Gaussian distribution functions of AE-index fluctuations: Evidence for time intermittency, Geophys. Res. Lett., 25, 4087-4090, 1998.

Consolini, G. and De Michelis, P.: Fractal time statistics of AEindex burst waiting times: evidence of metastability, Nonlin. Processes Geophys., 9, 419-423, doi:10.5194/npg-9-419-2002, 2002.

Consolini, G. and De Michelis, P.: Rank ordering multifractal analysis of the auroral electrojet index, Nonlin. Processes Geophys., 18, 277-285, doi:10.5194/npg-18-277-2011, 2011.

Consolini, G., Marcucci, M. F., and Candidi, M.: Multifractal Structure of Auroral Electrojet Index Data, Phys. Rev. Lett., 76, 40824085, 1996.

Dobias, P. and Wanliss, J. A.: Intermittency of storms and substorms: is it related to the critical behaviour?, Ann. Geophys., 27, 2011-2018, doi:10.5194/angeo-27-2011-2009, 2009. 
Keylock, C. J.: Characterizing the structure of nonlinear systems using gradual wavelet reconstruction, Nonlin. Processes Geophys., 17, 615-632, doi:10.5194/npg-17-615-2010, 2010.

Klimas, A. J., Vassiliadis, D. V., Baker, D. N., and Roberts, D. A.: The organized nonlinear dynamics of magnetosphere, J. Geophys. Res., 101, 13089-13114, 1996.

Klimas, A. J., Valdivia, J. A., Vassiliadis, D., Baker, D. N., Hesse, M., and Takalo, J.: Self-organized criticality in the substorm phenomenon and its relation to localized reconnection in the magnetosphere plasma sheet, J. Geophys. Res., 105, 18765-18780, 2000.

Kovács, P., Carbone, V., and Vörös, Z.: Wavelet-based filtering of intermittent events from geomagnetic time-series, Planet. Space Sci., 49, 1219-1231, 2001.

Mandelbrot, B. B. and Van Ness, J. W.: Fractional Brownian Motions, Fractional Noises and Applications, Soc. Indust. Appl. Math. Rev., 10, 422-437, 1968.

Muniandy, S. V. and Lim, S. C.: Modeling of locally selfsimilar processes using multifractional Browninan motion of Riemann-Liuouville type, Phys. Rev. E, 63, 046104, doi:10.1103/PhysRevE.63.046104, 2001.

Peltier, R. F. and Lévy-Vehel, J.: Multifractional Brownian motion: definition and preliminary results, INRIA Preprint No. 2645, 1995.

Peng, C. K., Buldryrev, S. V., Havlin, S., Simons, M., Stanley, H. E., and Goldberg, A. L.: Mosaic organization of DNA nucleotides, Phys. Rev. E, 49, 1685-1689, 1994.

Rambaldi, S. and Pinazza, O.: An accurate fractional Browninan motion generator, Physica A, 208, 21-30, 1994
Smith, S. W.: Digital filters, in: Digital Signal Processing: A prractical Guide for Engineers and Scientists, Elsevier Science, Burlington, MA, 261-343, 2003.

Uritsky, V. M. and Pudovkin, M. I.: Low frequency $1 / f$-like fluctuations of the AE-index as a possible manifestation of selforganized criticality in the magnetosphere, Ann. Geophys., 16, 1580-1588, doi:10.1007/s00585-998-1580-x, 1998.

Wanliss, J. A.: Fractal properties of SYM-H during quiet and active times, J. Geophys. Res., 110, A03202, doi:10.1029/2004JA010544, 2005.

Wanliss, J. A. and Dobias, P.: Space storms as a phase transition, J. Atmos. Sol. Terr. Phys., 69, 675-684, doi:10.1016/j.jastp.2007.01.001, 2007.

Wanliss, J. A. and Uristky, V.: Understanding bursty behavior in midlatitude geomagnetic activity, J. Geophys. Res., 34, L04107, doi:10.1029/2009JA014642, 2010.

Wanliss, J. A. and Weygand, J. M.: Power law burst lifetime distribution of the SYM-H index, Geophys. Res. Lett., 34, L04107, doi:10.1029/2006GL028235, 2007.

Wanliss, J., Anh, V. V., Yu, Z.-G., and Watson, S.: Multifractal modeling of magnetic storms via symbolic dynamics analysis, J. Geophys. Res., 110, A08214, doi:10.1029/2004JA010996, 2005.

Woodard, R., Newman, D. E., Sánchez, R., and Carreras, B. A.: Persistent dynamic correlations in self-organized critical systems away from their critical point, Physica A, 373, 215-230, doi:10.1016/j.physa.2006.05.001, 2007. 